\title{
Substrate Recognition by Novel Family of Amino Acid:[Carrier Protein] Ligases ${ }^{\dagger}$
}

\author{
Marko Močibob, ${ }^{a}$ Nives Ivić, ${ }^{b}$ Deni Šubašić, ${ }^{a, t}$ Marija Luić, ${ }^{b}$ and Ivana Weygand-Đuraševića,* \\ ${ }^{a}$ University of Zagreb, Faculty of Science, Department of Chemistry, Horvatovac 102a, 10000 Zagreb, Croatia \\ ${ }^{\mathrm{b}}$ Rudjer Boskovic Institute, Department of Physical Chemistry, Bijenicka 54, 10000 Zagreb, Croatia
}

RECEIVED DECEMBER 23, 2010; REVISED FEBRUARY 11, 2011; ACCEPTED FEBRUARY 14, 2011

\begin{abstract}
Amino acid:[carrier protein] ligases (aa:CP ligases) are newly discovered homologs of aminoacyl-tRNA synthetases (aaRS) which aminoacylate carrier proteins instead of tRNA. Activated amino acids are attached to prosthetic group of carrier proteins by thioester bond. Weak thioacylation activity was observed for many conventional aaRS. Therefore, different thiols were investigated as substrate analogs for aa:CP ligases. Here we show that coenzyme A, dithiothreitol and cysteine are efficiently aminoacylated by aa:CP ligases. The crystal structure of aa:CP ligase from Bradyrhizobium japonicum in complex with coenzyme A was solved, and revealed that CoA, besides acting as the substrate analog of the carrier protein, also competes with ATP for binding to the active site. aa:CP ligases do not aminoacylate tRNA, although they remarkably resemble catalytic core of atypical seryl-tRNA synthetases (aSerRS) from methanogenic archaea. Since aa:CP ligases lack tRNA-binding domain, fusion proteins of aa:CP ligases and aSerRS tRNA-binding domain were prepared in attempt to restore tRNA aminoacylation activity. Although fusion proteins were able to bind tRNA through appended domain, tRNA could not substitute carrier proteins in aminoacylation reaction. (doi: 10.5562/cca1818)
\end{abstract}

Keywords: aminoacyl-tRNA synthetase, amino acid:[carrier protein] ligase, carrier protein, coenzyme A, seryl-tRNA synthetase, thioester, tRNA

\section{INTRODUCTION}

Aminoacyl-tRNA synthetases are ancient and universal enzymes that catalyze the transfer of amino acids onto the appropriate transfer RNAs (tRNAs), primarily for use by the ribosome in protein synthesis. ${ }^{1}$ Because of their essential roles in translation of genetic code, genes encoding aaRSs appeared when life began. ${ }^{2,3}$ This long evolutionary history allowed the development of many other functions carried out by aaRSs, beyond their canonical role in protein biosynthesis.

The tRNA aminoacylation reaction catalyzed by canonical aaRSs proceeds in two steps. The amino acid (aa) is first activated at the expense of ATP, to form aminoacyl-adenylate (aa-AMP), which stays tightly bound to the enzyme, while inorganic pyrophosphate $\left(\mathrm{PP}_{\mathrm{i}}\right)$ is released:

$$
\text { aa }+ \text { ATP }+ \text { aaRS } \rightarrow \text { aaRS:aa-AMP }+ \text { PP }_{i}
$$

The activated amino acid is then transferred from the adenylate to the 3 '-end of the tRNA to form aminoacylated tRNA (aa-tRNA), in the reaction that releases AMP:

aaRS:aa-AMP + tRNA $\rightarrow$ aa-tRNA + AMP + aaRS

In the absence of aaRSs, amino acid activation reaction occurs at rate of only $\sim 8.3 \times 10^{-9} \mathrm{~mol}^{-1} \mathrm{~s}^{-1}$ (Refs. 4, 5) and has higher activation energy barrier than any reaction involved in translation of mRNA. Thus, the emergence of simple amino acid activating enzymes was crucial event in the evolution of protein synthesis machinery. aaRS family very likely evolved from simpler polypeptide chains by progressive accumulation of additional modular domains to the catalytic core with amino acid binding ability, allowing development of multi-domain, multi-functional contemporary enzymes of remarkable catalytic activity and specificity. Modern aaRSs can be divided into two classes, each consisting of $\sim 10$ members. ${ }^{6}$ Although they developed from different ancestors, and consequently share very little structural similarity, these two aaRS groups emerged

\footnotetext{
$\dagger$ This article belongs to the Special Issue Chemistry of Living Systems devoted to the intersection of chemistry with life.

* Present address: Institute of Molecular Life Sciences, University of Zurich, Winterthurerstrasse 190, Zurich, Switzerland and Molecular Life Sciences PhD program, Life Science Zurich Graduate School, ETH/University of Zurich, Zurich, Switzerland

* Author to whom correspondence should be addressed. (E-mail: weygand@chem.pmf.hr)
} 
simultaneously. ${ }^{7-9}$ In addition to two aaRS classes with housekeeping cellular functions, numerous gene duplication events have generated a broad group of proteins generally referred to as aaRS-like proteins. Some of them are homologous to the full-length aaRSs, although the majority represent molecules homologous with specific domain of aaRS, that may or may not carry out functions related to the aminoacylation of tRNA.

Seryl-tRNA synthetases are the enzymes responsible for the serylation of tRNA ${ }^{\text {Ser }}$ isoacceptors. They belong to subclass IIa of aaRSs, together with alanyl-, prolyl-, threonyl-, glycyl-, and hystidyl-tRNA synthetases. SerRSs can be further divided into two structurally different groups: bacterial-type SerRSs function in a variety of archaeal, bacterial and eukaryotic organisms, whereas the methanogenic-type was found only in methanogenic archaea. ${ }^{10,11}$ All seryl-tRNA synthetases (SerRSs) are functional homodimers with a C-terminal active site domain typical for class II aaRSs and an Nterminal domain that is responsible for tRNA binding. ${ }^{12-15}$ We have recently shown that methanogenictype SerRSs display several idiosyncratic structural features and exhibit a different mode of substrate recognition in comparison with bacterial-type SerRSs. ${ }^{15-17}$ Full-length or truncated SerRS-like proteins, homologous to both types of housekeeping SerRSs have been identified. SerRSs significantly divergent from canonical bacterial SerRSs, were found in two antibiotic producing Streptomyces species. In $S$. viridifaciens, the pathway-specific seryl-tRNA synthetase (VlmL) from valanimycin biosynthesis cluster catalyzes the formation of seryl-tRNA, from which the seryl residue is transferred to another substrate in the valanimycin biosynthetic pathway. ${ }^{18}$ A bacterial SerRS homolog (Alb10), which confers antibiotic resistance to the host cells, is encoded by the gene residing in a cluster required for albomycin synthesis in Streptomyces sp. strain ATCC 700974 (Ref 19). In both organisms housekeeping bacterial-type SerRS is encoded by the second serS gene at different genetic locus. A new seryl-tRNA synthetase-like protein (named SLIMP) with an essential mitochondrial function has been identified in insect. ${ }^{20}$ Although the exact mechanism of SLIMP action remains unclear, Guitart et al. found that lethal knock-out of SLIMP gene could be suppressed by supplementing insect diet with antioxidants, suggesting a role of SLIMP in oxidative stress. This SerRS paralog has lost the ability to bind amino acid and ATP, but retained tRNA binding. In contrast, we have recently described and characterized a new class of truncated SerRS-like proteins, which activate selected amino acids, but are deprived of the tRNA-binding domain and thus they lack canonical tRNA aminoacylating activity. ${ }^{21}$ Their genes were identified in numerous bacterial genomes. Expressed shortened SerRS-like proteins, which are structurally related to the catalytic core of atypical methanogenic-type SerRS (aSerRS), ${ }^{21}$ were shown to transfer several activated amino acids to the - $\mathrm{SH}$ of phosphopantetheine prosthetic group, covalently attached to the carrier proteins of yet unknown function. Therefore, these new enzymes act as amino acid:[carrier protein] ligases (aa:CP ligases). These SerRS-like proteins preferentially utilize glycine or alanine instead of serine as substrates in carrier protein aminoacylation reaction, although the overall mechanism of amino acid recognition and the architecture of the active site are largely preserved and resemble those in methanogenic-type SerRSs. Furthermore, it was shown that the amino acid recognition is relaxed and rather unspecific, in sharp contrast to exquisitely high specificity of conventional SerRS and aaRS in substrate recognition. In this work we explore the specificity of SerRS-like aa:CP ligases towards amino acid acceptors. Here we show that small molecular weight thiols, coenzyme A and dithiothreitol in particular, can substitute carrier proteins in aminoacylation reaction catalyzed by aa:CP ligases, but tRNA is not a substrate for aminoacylation even when the missing tRNA binding domain is appended to aa:CP ligases, to facilitate tRNA binding.

\section{EXPERIMENTAL}

\section{Preparation of Enzymes and tRNA}

Three aSerRS homologs (aa:CP ligases B110957 and Bll6282 from Bradyrhizobium japonicum and aa:CP ligase Atu2573 from Agrobacterium tumefaciens) were expressed as recombinant proteins in Escherichia coli. The proteins were His-tagged at $\mathrm{N}$-terminus and were purified by standard procedures on Ni-NTA agarose (Qiagen) as previously described. ${ }^{21}$ In-frame fusions of N-terminal tRNA binding domain from Methanosarcina barkeri SerRS and aa:CP ligase coding sequences were created by overlap PCR. ${ }^{21}$ PCR fragments coding for fusion proteins were cloned to $\mathrm{pET} 28 \mathrm{~b}$, and N-terminal His-tagged fusion proteins were overexpressed and purified as described. Preparation of SerRS from $E$. coli $^{22}$ and M. barkeri $^{23}$ was described previously.

Isolation of unfractioned tRNA from $B$. japonicum and $A$. tumefaciens was performed by phenol extraction and polyetilene-glycol precipitation. Crude unfractioned tRNA was deacylated and further purified by anion-exchange chromatography on DEAE-cellulose, essentially as described. ${ }^{21,24}$ Preparations of unfractioned tRNA from $A$. tumefaciens contained $4.6 \%$ tRNA $^{\text {Ser }}$ and $8.8 \%$ tRNA $^{\text {Ala }}$ isoacceptors, while $B$. japonicum bulk tRNA contained $5.4 \%$ tRNA $^{\text {Ser }}$ and $9.4 \%$ tRNA $^{\text {Gly }}$, as determined by aminoacylation with $E$. coli SerRS and $A$. tumefaciens or $B$. japonicum protein 
extracts. Unfractioned E. coli tRNA was purchased from Roche.

\section{ATP Hydrolysis Assay}

Thiol-dependent ATP hydrolysis was assayed at $30{ }^{\circ} \mathrm{C}$ in solution containing $50 \mathrm{mmol} \mathrm{\textrm {dm } ^ { - 3 }}$ TrisHCl $(\mathrm{pH}=7.5)$, $150 \mathrm{mmol} \mathrm{dm}^{-3} \mathrm{KCl}, \quad 0.2 \mathrm{mg} \mathrm{ml}^{-1}$ BSA, $5 \mathrm{mmol} \mathrm{dm}^{-3}$ $\mathrm{MgCl}_{2}, \quad 50 \mu \mathrm{mol} \mathrm{dm}^{-3} \quad\left[\alpha_{-}{ }^{32} \mathrm{P}\right]-\mathrm{ATP}, \quad 1 \mathrm{mmol} \mathrm{dm}^{-3}$ Ala (Atu2573) or $4 \mathrm{mmol} \mathrm{dm}^{-3}$ Gly (B110957, B116282), $10 \mathrm{U} / \mathrm{ml}$ inorganic pyrophosphatase (NEB), $0.6-1.2 \mu \mathrm{mol} \mathrm{dm}{ }^{-3}$ enzyme and $5 \mathrm{mmol} \mathrm{dm}^{-3}$ of different thiols (DTT, CoA, Cys or homocysteine). The reaction was stopped by mixing $2.5 \mu \mathrm{l}$ of reaction mixture with $3.75 \mu \mathrm{l}$ of solution containing $1 \mathrm{~g} \mathrm{dm}^{-3}$ SDS and $0.5 \mathrm{~mol} \mathrm{dm}{ }^{-3}$ sodium acetate $(\mathrm{pH}=5.0)$. Radiolabeled products, $\left[{ }^{32} \mathrm{P}\right]$-AMP and $\left[{ }^{32} \mathrm{P}\right]-$ ATP, were separated on PEI-cellulose TLC plates (SigmaAldrich) developed in $0.1 \mathrm{~mol} \mathrm{dm}{ }^{-3}$ ammonium acetate and 5\% acetic acid. Dried TLC plates were exposed on an imaging plate overnight, scanned using the Typhoon PhosphorImager (GE Healthcare), and quantified using ImageQuant 5.2 software (Molecular Dynamics).

\section{TLC Analysis of Thiol Aminoacylation}

Transfer of radiolabeled amino acids to CoA, DTT or Cys was monitored under similar conditions as described for ATP hydrolysis assay, except ATP and enzyme concen-

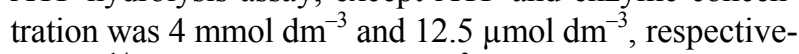

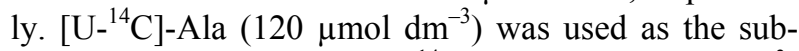
strate for Atu2573 and $\left[\mathrm{U}_{-}{ }^{14} \mathrm{C}\right]$-Gly $\left(200 \mu \mathrm{mol} \mathrm{dm}{ }^{-3}\right)$ was used as the substrate for B110957 and Bll6282. After 30 minutes of incubation, reaction mixtures were spotted directly to TLC plates. The products of CoA, DTT and Cys aminoacylation were separated by different TLC procedures. Reaction mixtures containing CoA were spotted to PEI-cellulose TLC plates (SigmaAldrich) and the plates were developed in solution containing $0.1 \mathrm{~mol} \mathrm{dm}^{-3}$ ammonium acetate, $0.2 \mathrm{~mol} \mathrm{dm}^{-3}$ $\mathrm{NaCl}$ and $5 \%$ acetic acid. DTT aminoacylation was analyzed on microcrystalline cellulose TLC plates (Fluka) developed with 2-propanol:formic acid:water 20:1:5 as the mobile phase, while products of Cys aminoacylation were also analyzed on microcrystalline cellulose TLC plates but developed with different solvent system (butanol:acetic acid:water 4:1:1). Radiolabeled products were visualized using Typhoon PhosphorImager.

\section{Crystallization and Structure Determination of Bll0957:CoA Complex}

Crystals of aa:CP ligase B110957 from B. japonicum were grown in a hanging drop by equilibrating $1 \mu \mathrm{l}$ of protein solution $\left(21 \mathrm{mg} \mathrm{ml}^{-1}\right)$ and $1 \mu \mathrm{l}$ of reservoir solution $\left(0.17 \mathrm{~mol} \mathrm{dm}^{-3}\right.$ ammonium acetate $(\mathrm{pH}=4.6)$, $0.085 \mathrm{~mol} \mathrm{dm}^{-3}$ sodium acetate, PEG $4000 w=25.5 \%$, glycerol $\varphi=15 \%$ ) as previously described. ${ }^{21}$ Obtained
Table 1. Data collection and refinement statistics for B110957:CoA complex

\begin{tabular}{lc}
\hline & Crystal \\
\hline Data collection & \\
Space group & $P 2_{1}{ }_{2} 2$ \\
Cell dimensions & \\
$a, b, c / \AA$ & $128.19,101.79,50.62$ \\
$\alpha, \beta, \gamma / \circ$ & $90,90,90$ \\
Resolution / $\AA$ & $20.86-2.20(2.32-2.20)^{*}$ \\
$R_{\text {merge }}$ & $0.075(0.444)$ \\
$I / \sigma I$ & $15.7(2.7)$ \\
Completeness / \% & $99.8(99.9)$ \\
Redundancy & $4.9(3.2)$ \\
& \\
Refinement & \\
Resolution / $\AA$ & $20.86-2.20$ \\
No. reflections & 32404 \\
$R_{\text {work }} / R_{\text {free }}$ & $0.1670 / 0.2228$ \\
No. atoms & 4996 \\
Protein & 4585 \\
Ligand/ion & $60 / 2$ \\
Water & 322 \\
$B$-factors & \\
Protein & 20.78 \\
Ligand/ion & $35.00 / 23.95$ \\
Water & 30.49 \\
R.m.s. deviations & \\
Bond lengths / $\AA$ & 0.007 \\
Bond angles / & 1.041 \\
\hline One crystal was used for data measurement. \\
* Values in parentheses are for highest-resolution shell. \\
\end{tabular}

crystals were then soaked for $3-5 \mathrm{~h}$ in a reservoir solution supplemented with $50 \mathrm{mmol} \mathrm{dm}^{-3} \mathrm{CoA}, 10 \mathrm{mmol} \mathrm{dm}^{-3}$ Gly, $10 \mathrm{mmol} \mathrm{dm}^{-3}$ ATP and $30 \mathrm{mmol} \mathrm{dm}^{-3} \mathrm{MgCl}_{2}$. Crystals were flash-frozen in liquid nitrogen, data was collected on in-house Xcalibur Nova R diffractometer (Oxford Diffraction), scaled and converted to structure factor intensities using Scala and Truncate, respectively, from the CCP4 program suite. ${ }^{25} \operatorname{Coot}^{26}$ was used to model substrates into the active site and Phenix.refine ${ }^{27}$ program for rigid body and restrained refinement. Data collection and refinement statistics are summarized in Table 1. The atomic coordinates and structure factors of Bl10957:CoA complex have been deposited in the Protein Data Bank (PDB ID code 3PZC).

\section{Inhibition of Amino Acid Activation by CoA}

Inhibition of amino acid activation by CoA was monitored in ATP-pyrophosphate exchange assay. ${ }^{28}$ ATP-PP $_{\mathrm{i}}$ exchange assay was performed at $30{ }^{\circ} \mathrm{C}$ in $100 \mathrm{mmol}$ $\mathrm{dm}^{-3}$ TrisHCl $(\mathrm{pH}=7.5), 150 \mathrm{mmol} \mathrm{dm} \mathrm{dm}^{-3} \mathrm{KCl}$,

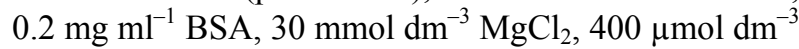
Gly, $1-2.5 \mu \mathrm{mol} \mathrm{dm}{ }^{-3}$ enzyme, $4 \mathrm{mmol} \mathrm{dm}^{-3}$ ATP and $1 \mathrm{mmol} \mathrm{dm}{ }^{-3}\left[{ }^{32} \mathrm{P}\right]-\mathrm{PP}_{\mathrm{i}}$ or $0.2 \mathrm{mmol} \mathrm{dm}^{-3} \mathrm{ATP}$ and $0.05 \mathrm{mmol} \mathrm{dm}^{-3}\left[{ }^{32} \mathrm{P}\right]-\mathrm{PP}_{\mathrm{i}}$, and varying concentrations of CoA (1-15 mmol dm $\left.{ }^{-3}\right)$. Reaction was quenched, products were separated on PEI-cellulose TLC plates devel- 

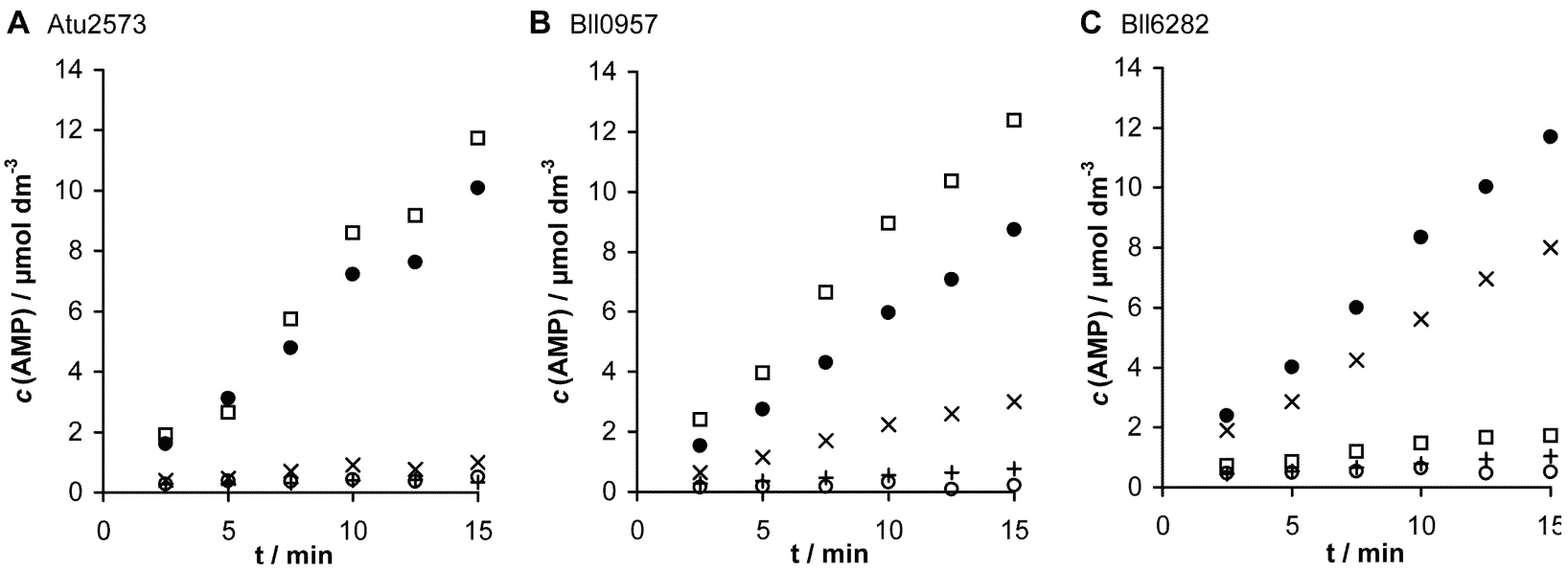

O no thiol $\square$ CoA $\bullet$ DTT $\times$ Cys + HomoCys

Figure 1. Thiol-dependent ATP hydrolysis by aa:CP ligases from Agrobacterium tumefaciens (Atu2573; panel A) and Bradyrhizobium japonicum (B110957 and B116282; panels B and C). The enzymes were incubated in the presence of dithiotreithol $(\bullet)$, coenzyme A ( $\square$ ) cysteine $(\times)$ or homocysteine $(+)$. No significant hydrolysis of ATP was observed in the absence of thiols $(\circ)$.

oped in $0.75 \mathrm{~mol} \mathrm{dm}^{-3} \mathrm{KH}_{2} \mathrm{PO}_{4}(\mathrm{pH}=3.5)$ and $4 \mathrm{~mol} \mathrm{dm}^{-3}$ urea and quantified by phosphorimaging, as described for ATP hydrolysis assay.

\section{tRNA Aminoacylation Assay}

Aminoacylation of tRNA was monitored by standard tRNA aminoacylation assay. ${ }^{28}$ Reaction was performed at $30{ }^{\circ} \mathrm{C}$ in $50 \mathrm{mmol} \mathrm{dm}^{-3}$ TrisHCl $(\mathrm{pH}=7.5)$, $150 \mathrm{mmol} \mathrm{dm}^{-3} \mathrm{KCl}, 0.4 \mathrm{mg} \mathrm{m}^{-1} \mathrm{BSA}, 15 \mathrm{mmol} \mathrm{dm}^{-3}$ $\mathrm{MgCl}_{2}, 5 \mathrm{mmol} \mathrm{dm}{ }^{-3}$ ATP, $150 \mu \mathrm{mol} \mathrm{dm}{ }^{-3}$ [U- $\left.{ }^{14} \mathrm{C}\right]-A l a$ (Atu2573), [U- $\left.{ }^{14} \mathrm{C}\right]-G l y ~\left(B 110957\right.$, Bll6282) or [U- $\left.{ }^{14} \mathrm{C}\right]-$ Ser (M. barkeri and E. coli SerRS), $2 \mathrm{mg} \mathrm{m}^{-1}$ of unfrac-

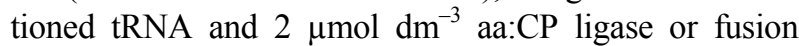
protein. Control reactions contained $0.5 \mathrm{mg} \mathrm{ml}^{-1}$ protein extract from $A$. tumefaciens or B. japonicum or $0.2 \mu \mathrm{mol} \mathrm{dm}{ }^{-3} M$. barkeri SerRS. Reaction mixtures were spotted on 3MM Whatman paper-discs, aminoacylated tRNA was precipitated with cold trichloroacetic acid $\left(100 \mathrm{~g} \mathrm{dm}^{-3}\right)$ and quantified by scintillation counting.

\section{RESULTS AND DISCUSSION}

\section{Thiol Aminoacylation by aa:CP Ligases}

The cognate substrates of aa:CP ligases are dedicated carrier proteins (CPs) carrying phosphopantetheine prosthetic group. ${ }^{21}$ Activated amino acids are transferred to sulfhydryl group of prosthetic arm and therefore aa:CP ligases catalyze thioester synthesis instead of tRNA esterification. It is well documented that many class $\mathrm{I}^{29-32}$ and class $\mathrm{II}^{33,34}$ aaRS, including E. coli SerRS, can transfer amino acids to different smallmolecular weight thiols, using either aminoacyl-tRNA or aminoacyl-adenylate as a donor. This thiol acylation activity is weak and it was interpreted as remnant edit- ing activity of ancient aaRSs, ${ }^{29-31}$ but also as a vestige from a thioester world in which ancestral aaRSs provided aminoacyl thioesters for noncoded peptide synthesis. ${ }^{32,34}$ Given that aa:CP ligases are by default devoted to thioester synthesis, and since they show obvious structural similarity to class II aaRS catalytic core, we wondered if small-molecular weight thiols could act as substrate analogs, i.e. amino acid acceptors, for aa:CP ligases.

Coenzyme A, dithiothreitol, cysteine and homocysteine were tested as substrates for aa:CP ligases Bl10957 and B1l6282 from B. japonicum and aa:CP ligase Atu2573 from A. tumefaciens. Thiol aminoacylation by aa:CP ligases was followed indirectly, by monitoring thiol-dependent ATP hydrolysis, indicative of catalytic turnover and concomitant thiol aminoacylation (Figure 1). Since aa:CP ligases Atu2573, Bll0957 and Bll6282 have different amino acid specificities ${ }^{21}$, thiol-

Table 2. Apparent rate constants (kapp) for thiol-dependent ATP hydrolysis by aa:CP ligases Atu2573, B110957 and Bl16282. ATP hydrolysis was assayed in the presence of $5 \mathrm{mmol} \mathrm{dm}{ }^{-3}$ coenzyme A (CoA), dithiotreithol (DTT), cysteine and homocysteine (HomoCys). The values are reported as arithmetic mean \pm standard error of mean (SEM).

\begin{tabular}{cccc}
\hline & \multicolumn{3}{c}{$k_{\text {app }} / 10^{-2} \mathrm{~s}^{-1}$} \\
\cline { 2 - 4 } & Atu2573 & B110957 & B116282 \\
\hline CoA & $2.1 \pm 0.2$ & $5.0 \pm 0.4$ & $0.26 \pm 0.01$ \\
DTT & $1.7 \pm 0.2$ & $3.16 \pm 0.05$ & $2.0 \pm 0.1$ \\
Cys & $0.15 \pm 0.03$ & $1.03 \pm 0.03$ & $1.31 \pm 0.09$ \\
HomoCys & n.d. & $0.212 \pm 0.007$ & $0.133 \pm 0.005$ \\
\hline
\end{tabular}


A

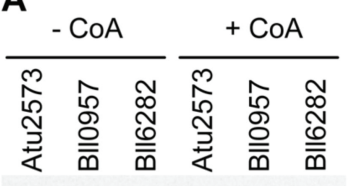

B

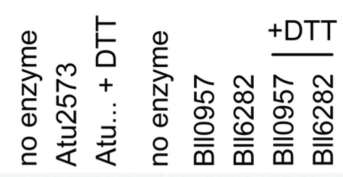

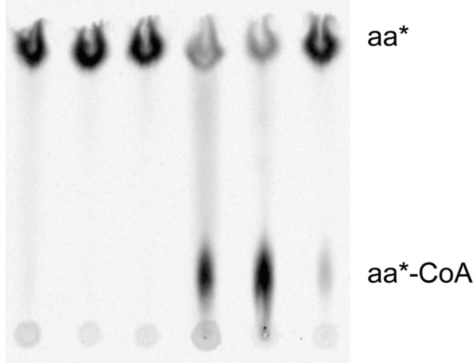

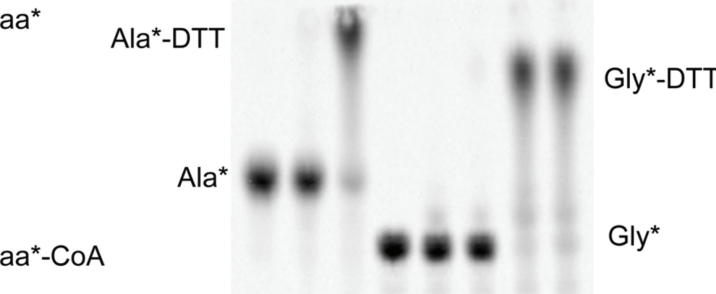

C

Figure 2. TLC analysis of thioester synthesis catalyzed by aa:CP ligases. In the presence of the coenzyme A (panel A), dithiotreitol (panel B) and cysteine (panel C), radiolabeled amino acids were converted into the new products by aa:CP ligases Atu2573, B110957 and B116282. $\left[{ }^{14} \mathrm{C}\right]$-Ala was used as substrate for Atu2573 and $\left[{ }^{14} \mathrm{C}\right]$-Gly for B110957 and B116282. The positions of radiolabeled amino acids and their products are indicated on the picture. In each case different TLC plates and solvent systems, optimized for particular separation, were used (see Experimental).

dependent ATP hydrolysis was followed in the presence of optimal amino acid substrate for particular enzyme: alanine in the case of Atu2573 and glycine in the case of B110957 and B116282. Curiously, enzymes display different preferences for thiols (Figure 1 and Table 2) as well: DTT is a good substrate for all three aa:CP ligases tested, CoA is the preferred substrate for B110957, while Atu2573 aminoacylates DTT and CoA approximately equally well. B1l6282 poorly aminoacylates CoA. Cys is aminoacylated by B1l6282 and B110957, but it is a weak substrate for Atu2573. Homocysteine was inefficiently recognized by all three enzymes tested. Apparent rate constants (Table 2) are comparable to and even exceed catalytic constants for thiol aminoacylation by different aaRS $\left(k_{\text {cat }}=0.2-1.8 \times 10^{-2} \mathrm{~s}^{-1}\right.$ for $\left.\mathrm{CoA}^{34}\right)$, as expected for enzymes optimized to catalyze thioester synthesis.

Apparent rate constants (Table 2) for aa:CP ligase aminoacylation of preferred thiol are close to turnover numbers for cognate CP aminoacylation by aa:CP ligases $\left(k_{\text {cat }}=0.35 \mathrm{~s}^{-1}\right.$ for B110957, $0.1 \mathrm{~s}^{-1}$ for B116282 and $0.64 \mathrm{~s}^{-1}$ for Atu2573) ${ }^{21}$; they are approximately only an order of magnitude lower than $k_{\text {cat }}$ in CP aminoacylation reaction. However, the apparent rate constants for thiol aminoacylation were measured under high substrate concentration ( $5 \mathrm{mmol} \mathrm{dm}{ }^{-3}$ ), unobtainable with cognate CPs. When CPs and thiols were assayed under identical conditions and much lower substrate concentration $\left(50 \mu \mathrm{mol} \mathrm{dm}{ }^{-3}\right)$, no enzymatic activity could be detected in the presence of the thiols. Therefore, small-molecular weight thiols act as substrate analogs of CPs, but only under high substrate concentration.

The transfer of activated amino acids to different thiols was confirmed by TLC analysis of the reaction mixtures containing ${ }^{14} \mathrm{C}$-amino acids (Figure 2). Examination of TLC plates revealed that radiolabeled amino acids were converted into the new products in the presence of the enzymes and various thiols. Different TLC plates and solvent systems were used to separate products of CoA, DTT and Cys aminoacylation in Figure 2 (see Experimental) and hence radiolabeled amino acids and their products have different mobility in Figure 2 $\mathrm{a}-\mathrm{c}$. The observed pattern of ${ }^{14} \mathrm{C}$-amino acid transformation on TLC plates mirrors the substrate preferences of different enzymes observed in the ATP hydrolysis assay (Figure 1): $\left[{ }^{14} \mathrm{C}\right]$-Gly is only partially converted in $S$-glycyl-CoA by Bll6282 (Figure 1c and Figure 2a). Bl10957 and Bll6282 convert $\left[{ }^{14} \mathrm{C}\right]-$ Gly into a new product (Figure 2c) in the presence of cysteine, but $\left[{ }^{14} \mathrm{C}\right]$-Ala remains unmodified by Atu2573, as expected from negligible stimulation of ATP hydrolysis by cysteine (Figure 1a).

\section{The Structure of Bl10957:CoA Complex}

aa:CP ligases and structurally related class II aaRSs aminoacylate fundamentally different macromolecular substrates: carrier proteins and tRNA, respectively. Amino acids are attached to the phosphopantetheine prosthetic group of carrier proteins by thioester bond. Coenzyme A also possesses phosphopantetheine moiety in its structure (Figure 3a) and it is efficiently recognized by aa:CP ligase B110957. We have recently solved the crystal structure of B110957 in complex with AMP, ATP and glycyladenylate analog. ${ }^{21}$ In order to gain the insight how the prosthetic group of carrier proteins is accommodated in aa:CP ligase active site, and to shed some light on the catalytic mechanism of thioester synthesis by aa:CP ligases, we prepared the B110957:CoA complex by soaking B110957 crystals in solution containing CoA.

The two polypeptide chains in the asymmetric unit form a homodimer. The overall structure closely resem- 

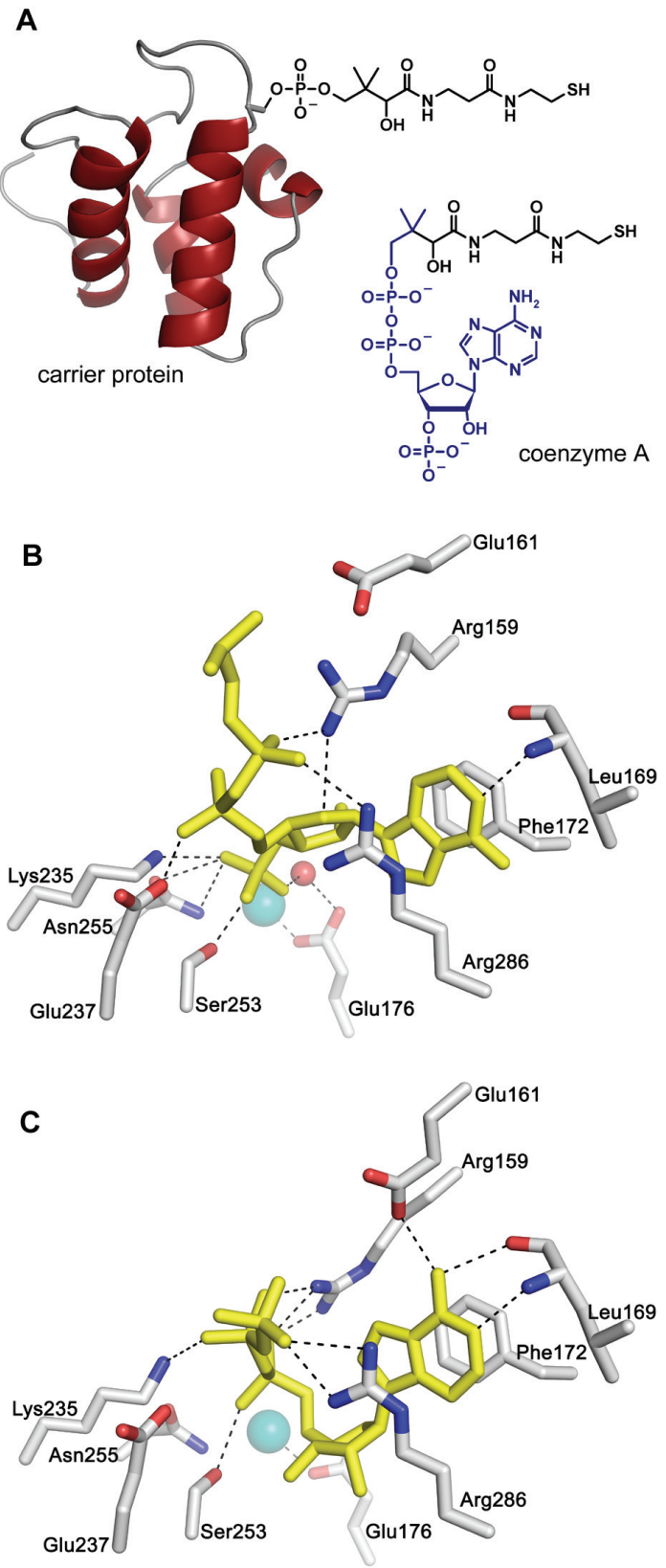

Figure 3. The complex of aa:CP ligase B110957 and CoA and its comparison to ATP complex. Panel A: schematic representation of carrier protein, the natural substrate of aa:CP ligases, and coenzyme A structure. The part of the CoA molecule visible in the electron density map of B110957:CoA complex (panel B) is colored blue. Panel B and C: CoA and ATP, respectively, bound in the active site of aa:CP ligase B110957. The ligands are colored yellow. Cyan sphere represents active site zinc ion. The red sphere is the water molecule. For clarity, some of the active site residues (e.g. Arg186) are not shown.

bles the catalytic core domain of methanogenic-type SerRS, ${ }^{16}$ as noted in our previous work. ${ }^{21}$ It comprises all three class II aaRS conserved motifs and zinc-ion characteristic for methanogenic-type SerRS. Binding of CoA molecule in the active site of B110957 (Figure 3b) caused no change in the overall structure (r.m.s.d. of $\mathrm{C} \alpha$ atoms between B110957:AMP and B110957:CoA is 0.26 $\AA$ ). CoA molecule is bound only in one subunit of the homodimer; which seems to be more disordered. The other active site is occupied with glycyl-adenylate, the natural reaction intermediate.

Coenzyme A resides in the part of the active site usually occupied with adenylate moiety of ATP or glycyl-adenylate. However, CoA binds to adenylate binding pocket in a notably different manner (Figure $3 \mathrm{~b}$ and $3 \mathrm{c})$. Adenine ring of $\mathrm{CoA}$ is bound in an inverted orientation compared to ATP. ${ }^{16,21}$ The orientation of CoA is also different from all to date known crystal structures of class II aaRS complexed with ATP or aminoacyladenylate intermediates. Nevertheless, the stacking interactions between conserved residues Phe172 and Arg286 are still maintained. The adenine ring of CoA forms putative hydrogen bonds with the main chain of Leu169, and via water molecules, with Arg173 and Asp285. The ribose moiety of the CoA molecule is dislocated compared to ATP, pointing its 3'-phosphate group towards the zinc atom. The closest distance between CoA and zinc-ion is $5.8 \AA$. This phosphate group is surrounded with Ser253, Lys235, Asn255 and even Glu176 (that coordinates zinc-ion) through a water molecule. Diphosphate moiety of CoA adopts almost the same position as $\beta$ - and $\gamma$ - phosphates of ATP molecule, making putative hydrogen bonds with Glu237 and arginines 159, 168 and 286. The pantetheine arm is completely disordered and could not be traced in the electron density maps indicating its high flexibility. Lack of strong interactions between the pantetheine moiety and the protein suggests that the recognition between Bl10957 and its carrier protein (i. e. natural macromolecular substrate) is not achieved through the protein-prosthetic group interactions but probably through protein-protein interactions. While most of the amino acid residues surrounding $\mathrm{CoA}$ are in the same position as in ATP binary structure, the side chain of Arg159 is oriented differently, pushing Glu161 side chain out from the active site. In the complex with ATP Arg159 is at hydrogen bond distance to $\alpha$ - and $\beta$ phosphates while side chain of Glu161 is hydrogen bonded to amino group of adenine ring.

The binding of CoA to aa:CP ligase B110957 in the absence of other substrates (ATP and amino acid) is dominated by interactions with nucleotide moiety of CoA instead of pantetheine arm. Pantetheine moiety of CoA was not resolved in the crystal structure, but it is clearly positioned away from the catalytic site. This suggests that the interaction with nucleotide moiety of CoA, differentiating CoA from genuine substrate (i. e. carrier protein), leads to misplacement of pantetheine moiety. 
The structure of aa:CP ligase B110957 in complex with $\mathrm{CoA}$ did not provide insight in prosthetic group accommodation and recognition, but instead revealed unanticipated binding mode of CoA to adenylate binding pocket. The structure of B110957:CoA complex indicated that $\mathrm{CoA}$, beside acting as $\mathrm{CP}$ substitute in aminoacylation reaction, can also act as ATP analog.

\section{CoA Inhibits Amino Acid Activation by aa:CP Ligase Bl10957}

To ascertain whether CoA could compete with ATP for binding to the active site, amino acid activation in ATPpyrophosphate exchange assay in the presence of $\mathrm{CoA}$ was investigated. ATP-PP ${ }_{\mathrm{i}}$ exchange assay monitors the first half of the overall aminoacylation reaction; amino acid activation for subsequent transfer of amino acid to appropriate acceptor. Formation of enzyme bound aminoacyl-adenylate intermediate results in incorporation of ${ }^{32} \mathrm{P}$ from $\mathrm{PP}_{\mathrm{i}}$ into ATP in the reverse reaction. CoA efficiently inhibited amino acid activation (Figure 4), confirming that CoA can compete with ATP for adenylate binding pocket. As expected for competitive inhibition, the extent of inhibition was dependent of ATP concentration (Figure 4): CoA concentration leading to $50 \%$ inhibition $\left(\mathrm{IC}_{50}\right)$ was $(5.0 \pm 0.5) \mathrm{mmol} \mathrm{dm}^{-3}$ in the presence of $0.2 \mathrm{mmol} \mathrm{dm}^{-3}$ ATP and $(23 \pm 3) \mathrm{mmol} \mathrm{dm}$ when the concentration of ATP was raised to $4 \mathrm{mmol} \mathrm{dm}^{-3}$. The same pattern of inhibition was observed with acetyl-coenzyme A, an analog of $\mathrm{CoA}$ incapable to accept activated amino acid, confirming that observed decrease of ATP-PP $\mathrm{P}_{\mathrm{i}}$ exchange is not the result of competition between $\mathrm{PP}_{\mathrm{i}}$ exchange and $\mathrm{CoA}$ aminoacylation reaction.

\section{tRNA Aminoacylation by aa:CP Ligases and Fusion Proteins with tRNA-Binding Domain}

An intriguing question is how aa:CP ligases emerged during evolution. They share ancestry with class II aaRS, atypical SerRS in particular, as evidenced by conserved signature motifs and common structural features. ${ }^{21}$ The main difference is the lack of N-terminal domain in aa:CP ligases, which plays the role of tRNAbinding domain in atypical SerRS. The aa:CP ligases might have evolved from modern aSerRS, by loss of tRNA binding domain and subsequent adaptation to a novel biological role - carrier protein aminoacylation. In that case, low level of residual tRNA acylation activity would be expected, and possibly tRNA aminoacylation activity could be restored by providing tRNA-binding domain in cis. It was demonstrated for AlaRS, ${ }^{35}$ MetRS $^{36}$ and SerRS ${ }^{37}$ that their catalytic domain devoid of tRNA-binding domain retains residual tRNA aminoacylation activity. On the other hand, aaRS are modular enzymes and it is generally accepted that modern

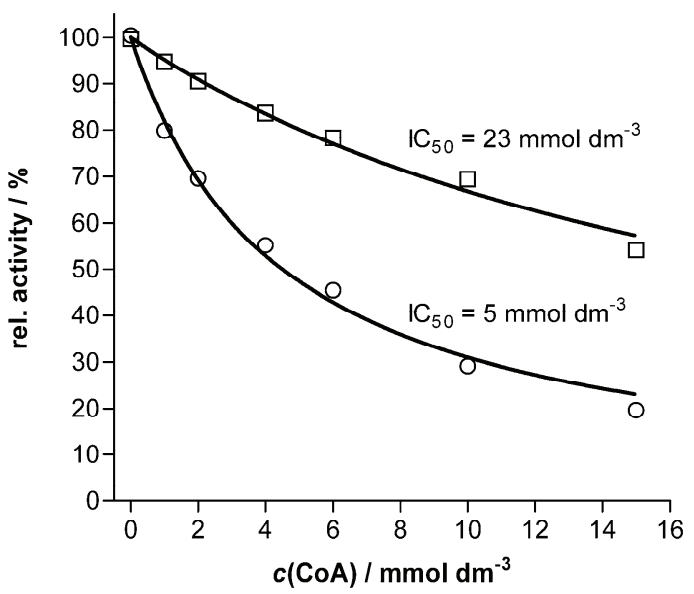

Figure 4. Inhibition of ATP-pyrophosphate exchange by coenzyme A. aa:CP ligase Bl10957 from Bradyrhizobium japonicum was incubated in the presence of $4 \mathrm{mmol} \mathrm{dm}^{-3}$ ATP and $1 \mathrm{mmol} \mathrm{dm}^{-3}$ pyrophosphate (口) or $0.2 \mathrm{mmol} \mathrm{dm}^{-3}$ ATP and $0.05 \mathrm{mmol} \mathrm{dm}^{-3}$ pyrophosphate $(\mathrm{O})$ and various concentrations of CoA. Calculated values of $\mathrm{CoA}$ concentrations leading to $50 \%$ inhibition $\left(\mathrm{IC}_{50}\right)$ are indicated on the graph.

aaRSs originated from primitive catalytic cores capable of amino acid activation by subsequent acquisition of additional domains, such as tRNA-binding and editing domains. ${ }^{9}$ Therefore, aa:CP ligases could have evolved from primordial class II aaRS catalytic core, before it acquired tRNA-binding domain and adopted tRNA aminoacylation activity. To explore these possibilities, we have challenged aa:CP ligases with various tRNA

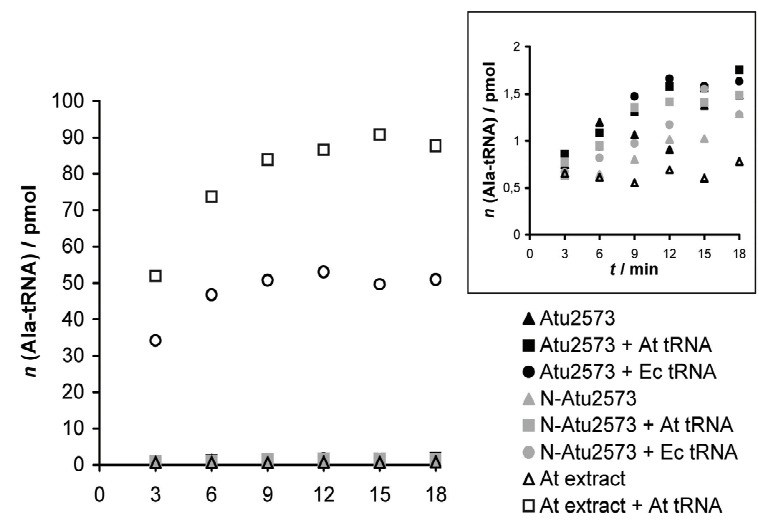

Figure 5. tRNA aminoacylation by aa:CP ligase Atu2573 from A. tumefaciens (black symbols) and its fusion protein with tRNA-binding domain from $M$. barkeri SerRS (N-Atu2573; gray symbols). The enzymes were assayed with unfractioned tRNA from $A$. tumefaciens (At tRNA; square symbols) and $E$. coli (Ec tRNA; round symbols) Triangles denote negative controls (no tRNA added). White symbols designate positive controls, tRNA aminoacylation by protein extract from A. tumefaciens. Insert: magnified view of the same data, expanded on the $\mathrm{y}$-axis. The same results were obtained with aa:CP ligases B110957 and Bll6282 from B. japonicum (not shown). 
substrates, and we have constructed fusion proteins of aa:CP ligases with N-terminal tRNA-binding domain from $M$. barkeri SerRS, in attempt to reveal their potentially cryptic tRNA acylation activity.

tRNA aminoacylation by aa:CP ligases was tested with various tRNA substrates, but in spite of many efforts no tRNA acylation activity could be detected (Figure 5). Homologous tRNA isolated from native source (i.e. $A$. tumefaciens for Atu2573, B. japonicum for Bl10957 and Bll6282) and heterologous tRNA from $E$. coli were offered to each enzyme. Unfractioned tRNA was used, in order to provide to enzymes all the naturally occurring tRNA isoacceptors. The tRNA isolated from $A$. tumefaciens and B. japonicum were efficiently aminoacylated in control reactions with protein extract prepared from the same organism, containing endogenous aaRSs, and also with purified E. coli SerRS, and thus they were competent substrates for aminoacylation reaction.

Fusion proteins were composed of tRNA-binding domain from $M$. barkeri aSerRS fused to N-terminus of Atu2573, B110957 and B116282. It is noteworthy that the aa:CP ligases, exemplified by the crystal structure of Bl10957, ${ }^{21}$ bear striking structural similarity to $M$. barkeri aSerRS and even possess the idiosyncratic HTH motif required for proper positioning of tRNA-binding domain in M. barkeri aSerRS. Fusion proteins were catalytically active in terms of amino acid activation, as verified by the active-site titration assay, ${ }^{28}$ but they were still unable to transfer activated amino acid to tRNA (Figure 5). M. barkeri SerRS, however, efficiently charges unfractioned tRNA from $A$. tumefaciens, $B$. japonicum and $E$. coli, in accordance with previous findings that aSerRS can aminoacylate tRNA ${ }^{\text {Ser }}$ from all three domains of life. ${ }^{38}$ Since tRNA-binding domain from $M$. barkeri SerRS can easily capture heterologous bacterial tRNA ${ }^{\text {Ser }}$ for charging by catalytic domain, the absence of aminoacylation activity of fusion proteins is not a consequence of cross-species barrier in tRNA recognition. To ascertain that the fusion proteins can capture tRNA by appendent tRNA-binding domain, formation of noncovalent protein:tRNA complexes was confirmed by electrophoretic mobility shift assay, although M. barkeri SerRS complexes with heterologous bacterial tRNA appeared more stable than the complexes of fusion proteins with tRNA (data not shown). These findings strengthen the conclusion that tRNA-binding domain can efficiently bind heterologous bacterial tRNA, although tRNA recognition is optimal in the native structural context of $M$. barkeri SerRS. In conclusion, tRNA can not substitute CPs in aminoacylation reaction by aa:CP ligases, even when the enzymes are endowed with tRNA-binding domain. However, lack of tRNA aminoacylation might be not due to inefficient tRNA recognition, but rather caused by the chemical nature of the reaction. It is possible that the aa: $\mathrm{CP}$ ligase active site is optimized for thioester synthesis, in such an extent that it cannot utilize -OH nucleophile as acceptor of activated amino acid. In this respect, it would be intriguing to replace 2'- and 3'-OH group of tRNA 3' terminal adenosine with thiol group, in attempt to alleviate potential "chemical barrier" in tRNA aminoacylation.

Appending of tRNA-binding domain to aa:CP ligases was insufficient to convert them to aminoacyltRNA synthetases and presumed cryptic tRNA aminoacylation activity could not be recuperated in fusion proteins. Although definitive proof is lacking, these findings favor the hypothesis that aa:CP ligases originated from ancient catalytic core capable of amino acid activation before it gained tRNA acylation activity and subsequently evolved into aaRS. This notion is further supported by relaxed amino acid specificity of aa:CP ligases, uncommon to contemporary aaRS. Thus, aa:CP ligases might represent a glimpse into the evolution of class II aaRS.

In this line it is worth mentioning that aa: $\mathrm{CP}$ ligases are not the only aaRS homologs which renounced tRNA aminoacylation. For example, MshC, a homolog of CysRS from Mycobacterium smegmatis transfers cysteine to carbohydrate precursor of mycothiol ${ }^{39}$ and recently described truncated homolog of LysRS ${ }^{40,41}$ aminoacylates elongation factor P. However, these homologs probably evolved from modern form aaRS, since MshC possesses shortened form of tRNA anticodon binding domain ${ }^{42}$ and has retained amino acid specificity for Cys, while elongation factor P mimics tRNA shape and negative charge and might be viewed as proteinaceus imitation of tRNA. ${ }^{43}$ It was proposed that asparagine synthetases, homologs of AsnRS lacking anticodon binding domain catalyzing asparagine synthesis, also originated in archaeal lineage from existing AspRS. ${ }^{44}$ In conclusion, the descendants of aaRS, once regarded as enzyme family faithfully devoted to tRNA aminoacylation and translation of genetic code, can transfer amino acids to surprisingly diverse acceptors of amino acids, ranging from inorganic ions $\left(\mathrm{NH}_{4}{ }^{+}\right)$, small organic molecules (mycothiol precursors) to macromolecules other than tRNA (CPs, elongation factor P).

\section{CONCLUSIONS}

aa:CP ligases can efficiently utilize CoA, DTT and Cys to a lesser extent as substrate analogs in aminoacylation reaction. The crystal structure of aa:CP ligase in complex with CoA reveals unusual mode of binding in ATP binding site. Contrary to our expectations, tRNA cannot substitute cognate carrier proteins in aminoacylation. No residual tRNA acylation activity could be recovered in aa:CP ligases even when they are fused with tRNAbinding domain which they originally lack. Therefore, 
aa:CP ligases diverged from aaRS ancestors before they acquired tRNA aminoacylation activity, and probably never served aminoacyl-tRNA synthesis and genetic code translation.

Coordinates. The atomic coordinates and structure factors of aa:CP ligase B110957 complexed with CoA have been deposited in the RCSB Protein Data Bank (www.pdb.org, PDB ID code 3pzc).

Acknowledgements. We are indebted to Morana Dulic for providing purified $M$. barkeri aSerRS. This work was supported by the grants from the Unity through Knowledge Fund (UKF, project 10/07) and the Ministry of Science, Education and Sports of the Republic of Croatia (projects 119-0982913-1358 and 098-11913442943).

\section{REFERENCES}

1. M. Ibba and D. Söll, Annu. Rev. Biochem. 69 (2000) 617-650.

2. C. W. Carter Jr, Annu. Rev. Biochem. 62 (1993) 715-748.

3. M. Guo, X.-L. Yang and P. Schimmel, Nat. Rev. Mol. Cell Biol. 11 (2010) 668-674.

4. A. J. Kirby and M. Younas, J. Chem. Soc. B 418 (1970) 11651172 .

5. Y. Pham, B. Kuhlman, G. L. Butterfoss, H. Hu, V. Weinreb and C. W. Carter Jr., J. Biol. Chem. 285 (2010) 38590-38601.

6. G. Eriani, M. Delarue, O. Poch, J. Gangloff and D. Moras, Nature 347 (1990) 203-206.

7. C. R. Woese, G. J. Olsen, M. Ibba and D. Söll, Microbiol. Mol. Biol. Rev. 64 (2000) 202-236.

8. P. O’Donoghue and Z. Luthey-Schulten, Microbiol. Mol. Biol. Rev. 67 (2003) 550-573.

9. L. Ribas de Pouplana and P. Schimmel, Cell. Mol. Life Sci. 57 (2000) 865-870.

10. H. S. Kim, U. C. Vothknecht, R. Hedderich, I. Celic and D. Söll, J. Bacteriol. 180 (1998) 6446-6449.

11. D. Tumbula, U. C. Vothknecht, H. S. Kim, M. Ibba, B. Min, T. Li, J. Pelaschier, C. Stathopoulos, H. Becker and D. Söll, Genetics 152 (1999) 1269-1276.

12. I. Weygand-Đurašević and S. Cusack, Seryl-tRNA Synthetases in: M. Ibba, C. Francklyn, S. Cusack (Eds.), The AminoacyltRNA Synthetases. Landes Biosciences, Georgetown (SAD), 2005, pp. 177-192.

13. S. Bilokapić, N. Ban and I. Weygand-Đurašević, Croat. Chem. Acta 82 (2009) 493-501.

14. S. Chimnaronk, M. Gravers Jeppesen, T. Suzuki, J. Nyborg and K. Watanabe, EMBO J. 24 (2005) 3369-3379.

15. J. Jarić, S. Bilokapić, S. Lesjak, A. Crnković, N. Ban and I. Weygand-Đurašević, J. Biol. Chem. 284 (2009) 30643-30651.

16. S. Bilokapić, T. Maier, D. Ahel, I. Gruić-Sovulj, D. Söll, I.
Weygand-Đurašević and N. Ban, EMBO J. 25 (2006) 2498-2509.

17. S. Bilokapić, N. Ivić, V. Godinić-Mikulčić, I. Piantanida, N. Ban and I. Weygand-Đurašević, J. Biol. Chem. 284 (2009) 10706 10713.

18. R. P. Garg, X. L. Qian, L. B. Alemany, S. Moran and R. J. Parry, Proc. Natl. Acad. Sci. U.S.A. 105 (2008) 6543-6547.

19. Y. Zeng, H. Roy, P. B. Patil, M. Ibba and S. Chen, Antimicrob. Agents. Chemother. 53 (2009) 4619-4627.

20. T. Guitart, T. L. Bernardo, J. Sagalés, T. Stratmann, J. Bernués and L. Ribas de Pouplana, J. Biol. Chem. 285 (2010) $38157-$ 38166.

21. M. Močibob, N. Ivić, S. Bilokapić, T. Maier, M. Luić, N. Ban and I. Weygand-Đurašević, Proc. Natl. Acad. Sci. U.S.A. 107 (2010) 14585-14590.

22. D. Ahel, D. Slade, M. Močibob, D. Söll and I. WeygandĐurašević, FEBS Lett. 579 (2005) 4344-4348.

23. D. Korenčić, C. Polycarpo, I. Weygand-Đurašević and D. Söll, J. Biol. Chem. 279 (2004) 48780-48786.

24. M. Močibob and I. Weygand-Đurašević, Arch. Biochem Biophys. 470 (2008) 129-138.

25. Collaborative Computational Project, Number 4, Acta Crystallogr. Sect. D 50 (1994) 760-763.

26. P. Emsley and K. Cowtan, Acta Crystallogr. Sect. D 60 (2004) 2126-2132.

27. V. Yu Lunin and T. P. Skovoroda, Acta Crystallogr. Sect. A 51 (1995) 880-887.

28. C. S. Francklyn, E. A. First, J. J. Perona and Y.-M. Hou, Methods 44 (2008) 100-118.

29. H. Jakubowski, Nucleic Acids Res. 23 (1995) 4608-4615.

30. H. Jakubowski, Nucleic Acids Res. 24 (1996) 2505-2510.

31. H. Jakubowski, Biochemistry 35 (1996) 8252-8259.

32. H. Jakubowski, J. Biol. Chem. 275 (2000) 34845-34848.

33. H. Jakubowski, Biochemistry 36 (1997) 11077-11085.

34. H. Jakubowski, Biochemistry 37 (1998) 5147-5153.

35. D. D. Buechter and P. Schimmel 32 (1993) 5267-5272.

36. D. Kohda, S. Yokoyama and T Miyazawa, J. Biol. Chem. 262 (1987) 558-563.

37. F. Borel, C. Vincent, R. Leberman and M. Härtlein, Nucleic Acids Res. 22 (1994) 2963-2969.

38. S. Bilokapić, D. Korenčić, D. Söll and I. Weygand-Đurašević, Eur. J. Biochem. 271 (2004) 694-702.

39. D. Sareen, M. Steffek, G. L. Newton and R. C. Fahey, Biochemistry 41 (2002) 6885-6890.

40. W. W. Navarre, S. B. Zou, H. Roy, J. L. Xie, A. Savchenko, A. Singer, E. Edvokimova, L. R. Prost, R. Kumar, M. Ibba and F. C. Fang, Mol. Cell 39 (2010) 209-221.

41. T. Yanagisawa, T. Sumida, R. Ishii, C. Takemoto and S. Yokoyama, Nat. Struct. Mol. Biol. 17 (2010) 1136-1143.

42. L. W. Tremblay, F. Fan, M. W. Vetting and J. S. Blanchard, Biochemistry 47 (2008) 13326-13335.

43. K. Hanawa-Suetsugu, S. Sekine, H. Sakai, C. Hori-Takemoto, T. Terada, S. Unzai, J. R. H. Tame, S. Kuramitsu, M. Shirouzu and Shigeyuki Yokoyama, Proc. Natl. Acad. Sci. U.S.A. 101 (2004) 9595-9600.

44. H. Roy H, H. D. Becker, J. Reinbolt and D. Kern, Proc. Natl. Acad. Sci. U.S.A. 100 (2003) 9837-9842. 